\title{
Discriminação racial no âmbito dos serviços de saúde: revisão das políticas públicas
}

\author{
Racial discrimination in health services: review of public policies \\ Discriminación racial en los servicios de salud: revisión de políticas públicas
}

Recebido: 01/08/2021 | Revisado: 04/08/2021 | Aceito: 06/08/2021 | Publicado: 18/08/2021

\author{
Francisco Eduardo Viana Brito \\ ORCID: https://orcid.org/0000-0001-9012-2914 \\ Centro Universitário Uninovafapi, Brasil \\ E-mail: eduardobritoenf@gmail.com \\ Hugo Alexandre Santos Silvério \\ ORCID: https://orcid.org/0000-0002-9943-4313 \\ Centro Universitário Uninovafapi, Brasil \\ E-mail: hugosilverio89@hotmail.com \\ Rafael Rodrigues Oliveira Coelho \\ ORCID: https://orcid.org/0000-0001-5367-1471 \\ Centro Universitário Uninovafapi, Brasil \\ E-mail: rafaelcoelho30@gmail.com \\ Marta Maria da Silva Lira Batista \\ ORCID: https://orcid.org/0000-0001-5995-0535 \\ Hospital Universitário do Piaú, Brasil \\ E-mail: fgamarthalira@gmail.com
}

\begin{abstract}
Resumo
A discriminação pode ser definida como um resultado comportamental ao estigma e preconceito, formando assim uma clara distinção entre ideias, atitudes ou ideologias, e suas consequências no comportamento discriminatório. $\mathrm{O}$ objetivo deste trabalho é suscitar à reflexão sobre discriminação racial no âmbito dos serviços de saúde bem como as práticas realizadas para minimização desta situação no Brasil. O presente estudo trata-se de uma revisão narrativa, retrospectiva e de caráter qualitativo. Foram examinadas publicações dos últimos 5 anos. A literatura científica publicada é indexada em uma variedade de bancos de dados. Os dados foram extraídos de bancos de dados para estudos. O racismo institucional existe na rotina de trabalho e na prática de profissionais da saúde e prestadores de serviços e se manifesta de forma excludente, ignorante e preconceituosa com base em normas e práticas predeterminadas e admitidas, constituindo-se um obstáculo de para o alcance dos direitos da população negra aos quais the são cabíveis pelas instituições. A nível institucional, o Governo no Brasil, tem desenvolvido algumas estratégias para minimizar o estigma e a discriminação na atenção básica, facilitando o acesso a saúde por grupos marginalizados, em uma tentativa de reduzir a morbimortalidade ligada a esses determinantes.
\end{abstract}

Palavras-chave: Grupos étnicos; Preconceito; Saúde das minorias étnicas; Grupos minoritários.

\begin{abstract}
Discrimination can be defined as a behavioral result of stigma and prejudice, thus forming a clear distinction between ideas, attitudes or ideologies, and their consequences on discriminatory behavior. The objective of this work is to raise reflection on racial discrimination within the scope of health services, as well as the practices carried out to minimize this situation in Brazil. The present study is a narrative, retrospective and qualitative review. Publications from the last 5 years were examined. Published scientific literature is indexed in a variety of databases. Data were extracted from databases for studies. Institutional racism exists in the work routine and practice of health professionals and service providers and manifests itself in an excluding, ignorant and prejudiced way based on predetermined and accepted norms and practices, constituting an obstacle to the achievement of rights of the black population to which they are entitled by the institutions. At the institutional level, the Government in Brazil has developed some strategies to minimize stigma and discrimination in primary care, facilitating access to health care for marginalized groups, in an attempt to reduce morbidity and mortality linked to these determinants.
\end{abstract}

Keywords: Ethnic groups; Prejudice; Health of ethnic minorities; Minority groups.

\section{Resumen}

La discriminación se puede definir como un resultado conductual del estigma y los prejuicios, formando así una clara distinción entre ideas, actitudes o ideologías y sus consecuencias sobre el comportamiento discriminatorio. El objetivo de este trabajo es suscitar la reflexión sobre la discriminación racial en el ámbito de los servicios de salud, así como las prácticas realizadas para minimizar esta situación en Brasil. El presente estudio es una revisión narrativa, retrospectiva y cualitativa. Se examinaron las publicaciones de los últimos 5 años. La literatura científica publicada está indexada en una variedad de bases de datos. Los datos se extrajeron de las bases de datos para los estudios. El 
racismo institucional existe en la rutina y práctica laboral de los profesionales de la salud y prestadores de servicios y se manifiesta de manera excluyente, ignorante y prejuiciosa basada en normas y prácticas predeterminadas y aceptadas, constituyendo un obstáculo para el logro de los derechos de la población negra a la que tienen derecho a las instituciones. A nivel institucional, el Gobierno de Brasil ha desarrollado algunas estrategias para minimizar el estigma y la discriminación en la atención primaria, facilitando el acceso a la atención de salud de los grupos marginados, en un intento por reducir la morbilidad y la mortalidad vinculadas a estos determinantes.

Palabras clave: Grupos étnicos; Prejuicio; Salud de las minorías étnicas; Grupos minoritarios.

\section{Introdução}

A discriminação pode ser definida como um resultado comportamental ao estigma e preconceito, formando assim uma clara distinção entre ideias, atitudes ou ideologias, e suas consequências no comportamento discriminatório. Portanto, a discriminação é uma relação de poder com os indivíduos de um determinado grupo social, esta relação os exclui e lhes atribui características de menor valor moral (Massignam et al., 2015).

Estudos a nível nacional e internacional mostram que existem discrepâncias importantes entre a saúde de brancos e negros, homens e mulheres, demonstrando interações simultâneas entre desigualdades sociais, raciais e de gênero em diversos locais e contextos, como educação, cinema e ocupacionais (Ashikhmin et al., 2017; Cave et al., 2020; Chen \& Mallory, 2021; Holloway \& Varner, 2021; Kalckmann et al., 2007; Martins, 2021; Modeling et al., 2019).

No Brasil, a partir de 1980, com o restabelecimento dos princípios da democracia, justiça social e igualdade, tem se refletido mais sobre a discriminação no campo da saúde pública e novos avanços teóricos e metodológicos têm sido feitos (Massignam et al., 2015).

A questão da discriminação nos serviços de saúde tem impulsionado diversas personalidades em diferentes regiões do mundo. Mesmo que primeiramente movido por algumas formas específicas de discriminação, como raça, esse movimento foi mais recentemente impulsionado por vários problemas e características identitárias (Bastos, 2015).

O objetivo deste trabalho é suscitar à reflexão sobre discriminação racial no âmbito dos serviços de saúde bem como as práticas realizadas para minimização desta situação no Brasil.

\section{Metodologia}

O presente estudo trata-se de uma revisão narrativa, retrospectiva e de caráter qualitativo. Foram examinadas publicações dos últimos 5 anos. A literatura científica publicada é indexada em uma variedade de bancos de dados. Os dados foram extraídos de bancos de dados para estudos. Cabe ressaltar que vários bancos de dados foram pesquisados para garantir que a maioria dos estudos relevantes foram identificados. Os bancos selecionados foram PubMed, Scielo, Science Direct, PsycINFO, CINAHL e matéria cinzenta.

Após encontrar um artigo relevante foram examinados os tópicos semelhantes, que fossem pertinentes à sua pergunta de revisão. Sequencialmente os resumos e artigos foram revisados a fim de eliminar todas as duplicatas. Ao final, as informações relevantes foram sintetizadas, integrando-as à redação, conforme apropriado.

\section{Resultados e Discussão}

O racismo institucional existe na rotina de trabalho e na prática de profissionais da saúde e prestadores de serviços e se manifesta de forma excludente, ignorante e preconceituosa com base em normas e práticas predeterminadas e admitidas, constituindo-se um obstáculo de para o alcance dos direitos da população negra aos quais the são cabíveis pelas instituições (Miskolci \& Pereira, 2019; Tavares \& Kuratani, 2019).

No campo da saúde, o racismo institucional existe em vários aspectos, desde a negação em reconhecer as 
peculiaridades da população negra e as diferenças de acometimento de doenças entre as raças até a ignorância, falta de discussão e profissionalização dos profissionais de saúde sobre questões raciais. Além das atitudes racistas que estão relacionadas às atividades profissionais, há também uma percepção de um sentimento de superioridade envolvido, que incluem ações como olhares diferentes, o silêncio e o desinteresse pela assistência (Tran et al., 2021).

Independentemente da recente promulgação de leis antidiscriminação e da melhoria da imagem do negro, seu real status na sociedade brasileira ainda é depreciativo. No campo governamental, observou-se que o alcance das iniciativas de eliminação da desigualdade racial ainda é limitado e é mais fácil de constatar em documentos e recomendações do que em ações reais (Dal Pai et al., 2018).

Portanto, pode-se considerar que a sociedade brasileira contemporânea ainda é racista e esse racismo também existe no Estado, e como efeito, nas instituições governamentais (Broca et al., 2020). A análise de situações percebidas como discriminatórias revela que as atitudes parecem estar ligadas à ideologia do dominador que perpassa o cotidiano estando introjetada nos profissionais, como, por exemplo, acreditar que as pessoas negras são "mais fortes e resistentes à dor", tratá-las com displicência, com uma tendência a minimizar as queixas (Moreira, n.d.).

Associar o tom da pele à baixa renda parece ser algo natural. Além disso, inúmeros relatos mostram que quando as pessoas estão mais vulneráveis, como durante a gravidez e o parto, a discriminação é mais frequente. Embora os relatos sejam relativamente menores, a literatura também aponta o racismo institucional entre gestores e trabalhadores, entre trabalhadores e entre usuários e trabalhadores negros. Muitos profissionais deixam de ser admitidos porque são negros, e cargos de direção e de alto escalão são preenchidos por pessoas brancas (Li et al., 2021; Tavares \& Kuratani, 2019).

Segundo a literatura, profissionais e gestores de saúde que não possuem experiência em lidar com a população negra podem ser obstáculos no acesso a saúde, reiterando a discrepância existente caracterizada por fatores socioeconômicos, classe social e etnia (Dal Pai et al., 2018; Miskolci \& Pereira, 2019; Tran et al., 2021).

Nesse caso, mostra-se relevante tratar sobre a saúde dessa parcela da população durante a formação dos profissionais, pois tal implementação melhoraria muito as condições de vida desse público por meio de ações em todos os níveis de atenção à saúde e do respeito as necessidades individuais (Conceição \& Corsino, 2021). Além de permitir que as pessoas recebam educação, que haja pesquisas sobre o tema e que esses cidadãos alcancem a gestão participativa dos serviços.

Na tentativa de minimizar a discriminação étnica e racial ao nível dos serviços de saúde, foi criada a Política Nacional de Saúde Integral da População Negra (PNSIPN) que consiste em uma série de medidas para o combate às desigualdades no Sistema único de Saúde (SUS) e para a promoção da saúde da população negra de forma integral.

A PNSIPN, mesmo que muitas vezes seja considerada algo desnecessário por alguns profissionais da atenção básica, possui caráter transversal e considera que as desigualdades em saúde são resultados de injustos processos socioeconômicos e culturais, como o vigente racismo, que corroboram com a morbimortalidade das populações negras brasileiras. Reconhecendo que se trata de uma ferramenta de prevenção ao racismo, deve-se entender que sua desconstrução requer o desenvolvimento de estratégias intersetoriais, principalmente envolvendo saúde e educação, para fortalecer o controle social e transformar as relações sociais discriminatórias de forma mais abrangente(Dal Pai et al., 2018; Kalckmann et al., 2007).

Considerando todos os aspectos relacionados à saúde pública e a efetividade dos princípios do Sistema Único de Saúde (SUS) para a população negra, é importante compreender como esses profissionais percebem o racismo institucional e as ações que eles realizam contra o racismo em diversas situações(Broca et al., 2020; Dal Pai et al., 2018; Miskolci \& Pereira, 2019).

É notório que a população negra vem sendo discriminada nos serviços de saúde, tanto como usuários, quanto como profissionais. Não se pode negar ainda, que a vivência da discriminação racial interfere sobremaneira na construção das identidades e na produção dos sujeitos, e consequentemente na saúde das pessoas (Li et al., 2021; Tavares \& Kuratani, 2019). 
Levando-se em consideração que um dos princípios básicos do SUS é a eqüidade, os serviços de saúde devem oferecer tratamentos diferenciados e específicos para os desiguais, visando reduzir diferenças de vulnerabilidade das populações (Kalckmann et al., 2007; Miskolci \& Pereira, 2019).

Tendo em vista a complexidade e dificuldade de mudança de uma estrutura social no curto prazo, além de intervenções estruturais e culturais, é necessário investir no nível institucional para estimular o estabelecimento de canais de comunicação entre usuários e gestores e proporcionar qualificação profissional para os trabalhadores para serem discutidas as conexões entre diferentes situações de discriminação e desigualdade social e as formas de lidar com essas desigualdades (Bastos \& Garcia, 2015; Dal Pai et al., 2018; Kalckmann et al., 2007; Massignam et al., 2015; Tavares \& Kuratani, 2019).

\section{Considerações Finais}

Felizmente, a nível institucional, o Governo no Brasil, tem desenvolvido algumas estratégias para minimizar o estigma e a discriminação na atenção básica, facilitando o acesso a saúde por grupos marginalizados, em uma tentativa de reduzir a morbimortalidade ligada a esses determinantes.

Entretanto, é válido ressaltar que essas políticas só se põem em prática quando são trazidas à realidade local específica, demandando um empenho coletivo, ou seja, com o apoio dos gestores, técnicos, conselheiros e demais envolvidos na produção do cuidado em saúde, assim como de pesquisadores e ativistas sociais comprometidos com a construção de uma sociedade mais justa.

\section{Referências}

Ashikhmin, A. V., Shishelova, A. Y., \& Aliev, R. R. (2017). tDCS provokes sustainable changes in EEG and reorganizes autonomic modulation of heart rate. Brain Stimulation, 10(2), 484-486. https://doi.org/10.1016/j.brs.2017.01.421

Bastos, J. L., \& Garcia, L. P. (2015). Discriminação nos serviços de saúde. Epidemiologia e Serviços de Saúde, 24(3), 352-351. https://doi.org/10.5123/s167949742015000300001

Broca, P. V., Rezende, J., \& Medeiros, M. (2020). Gerenciamento Das Situações De Violência No Trabalho Na. 1-14.

Campos, A. G. de O. et al. (2021). Prejudice and discrimination suffered by transgender persons in health services: Integrative literature review. Research, Society and Development, 10(5), e26510514590. https://doi.org/10.33448/rsd-v10i5.14590

Cave, L., Cooper, M. N., Zubrick, S. R., \& Shepherd, C. C. J. (2020). Racial discrimination and child and adolescent health in longitudinal studies: A systematic review. Social Science and Medicine, 250(May 2019), 112864. https://doi.org/10.1016/j.socscimed.2020.112864

Conceição, WL da., \& Corsino, LN. (2021). Educação e Justiça Social: apresentação. Pesquisa, Sociedade e Desenvolvimento, 10 (8). Obtido em https://rsdjournal.org/index.php/rsd/article/view/17878

Chen, S., \& Mallory, A. B. (2021). The effect of racial discrimination on mental and physical health: A propensity score weighting approach. Social Science \& Medicine, 285(July), 114308. https://doi.org/10.1016/j.socscimed.2021.114308

Chor, D., \& Lima, C. R. de A. Aspectos epidemiológicos das desigualdades raciais em saúde no Brasil. Cadernos de Saúde Pública, 21, $1586-1594$.

Dal Pai, D., Sturbelle, I. C. S., Santos, C. Dos, Tavares, J. P., \& Lautert, L. (2018). Violência física e psicológica perpetrada no trabalho em saúde. Texto e Contexto Enfermagem, 27(1), 1-10. https://doi.org/10.1590/0104-07072018002420016

Holloway, K., \& Varner, F. (2021). Forms and frequency of vicarious racial discrimination and African American parents' health. Social Science \& Medicine, July, 114266. https://doi.org/10.1016/j.socscimed.2021.114266

Kalckmann, S., Santos, C. G. dos, Batista, L. E., \& Cruz, V. M. da. (2007). Racismo institucional: um desafio para a eqüidade no SUS? Saúde e Sociedade, 16(2), 146-155. https://doi.org/10.1590/s0104-12902007000200014

Li, J. Y., Lee, Y., Tian, S., \& Tsai, W. (2021). Coping with workplace racial discrimination: The role of internal communication. Public Relations Review, 47(4), 102089. https://doi.org/10.1016/j.pubrev.2021.102089

Martins, B. S. (2021). Violência ocupacional na Enfermagem Occupational Violence in Nursing Violencia ocupacional en enfermería. $2021,1-14$. Massignam, F. M., Bastos, J. L. D., \& Nedel, F. B. (2015). Discriminação e saúde: um problema de acesso. Epidemiologia e Serviços de Saúde, 24(3), 544541. https://doi.org/10.5123/s1679-49742015000300020

Miskolci, R., \& Pereira, P. P. G. (2019). Educação e Saúde em disputa: movimentos anti-igualitários e políticas públicas. Interface - Comunicação, Saúde, Educação, 23, 1-14. https://doi.org/10.1590/interface.180353 
Research, Society and Development, v. 10, n. 10, e320101018947. 2021

(CC BY 4.0) | ISSN 2525-3409 | DOI: http://dx.doi.org/10.33448/rsd-v10i10.18947

Modeling, L. M., Measurement, F., Snowrift, O. N., Environmental, A. R., Regional, S., Power, E., Limited, G. C., Influence, T. H. E., Snow, O. F., On, F., Around, S., Embankment, T. H. E., Wind, I. N., Snow, B., Aspect, O. F., On, R. R., Distribution, S., Model, A. A. C., Simulation, N., ... Maciejowski, J. M. (2019). No 主観的健康感を中心とした在宅高齢者における 健康関連指標に関する共分散構造分析Title. Journal of Wind Engineering and Industrial Aerodynamics, 26(3), 1-4. https://doi.org/10.1007/s11273-020-09706-3\%0Ahttp://dx.doi.org/10.1016/j.jweia.2017.09.008\%0Ahttps://doi.org/ 10.1016/j. energy.2020.117919\%0Ahttps://doi.org/10.1016/j.coldregions.2020.103116\%0Ahttp://dx.doi.org/10.1016/j.jweia.2010.12.004\%0Ahttp://dx.doi.org/10.1016

Moreira, M. (n.d.). Pra nem morta ser calada : arte afro- brasileira como fortalecimento identitário entre estudantes de medicina. 441-450. https://doi.org/10.1590/0103-1104202112915

Tavares, J. S. C., \& Kuratani, S. M. de A. (2019). Manejo Clínico das Repercussões do Racismo entre Clinical Management of the Repercussions of Racism among Women who have "Turned Black" Introdução. 1-13.

Tran, N., Yabes, K., \& Miller, A. (2021). How should clinicians help patients navigate "Model minority" demands? AMA Journal of Ethics, 23(6), E456E464. https://doi.org/10.1001/AMAJETHICS.2021.456 